\title{
1 Bis(pinacolato)diboron as an additive for the detection of 2 thermal neutrons in plastic scintillators
}

3 Adam Mahl*, ${ }^{2}$ Henok A. Yemam*, ${ }^{1}$ John Stuntz, ${ }^{1}$ Tyler Remedes, ${ }^{2}$ Alan Sellinger, ${ }^{1}$ Uwe Greife ${ }^{2,+}$

4 1. Department of Chemistry and Geochemistry and the Materials Science Program Colorado School

5 of Mines, Golden, CO 80401, USA

6 2. Department of Physics and the Nuclear Science and Engineering Center (NuSEC), Colorado

$7 \quad$ School of Mines, Golden, CO 80401, USA

8 *Authors Adam Mahl and Henok A. Yemam contributed equally to this publication and should be 9 regarded as first authors.

10 + Corresponding author: ugreife@mines.edu

Abstract

A readily available and inexpensive boron compound was tested as an additive for the detection of thermal neutrons in plastic scintillators. Bis(pinacolato)diboron $\left(\mathrm{B}_{2} \mathrm{Pin}_{2}\right)$ was determined to be a compatible boron source $\left(8.51 \mathrm{wt} \%\right.$ boron, $\left.1.70 \mathrm{wt} \%{ }^{10} \mathrm{~B}\right)$ in poly(vinyltoluene) based matrices. Plastic scintillator blends of 1-20 wt\% 2,5-diphenyloxazole (PPO), $0.1 \mathrm{wt} \%$ 1,4-bis(5phenyloxazol-2-yl) benzene (POPOP) and 1-15 wt\% $\mathrm{B}_{2} \mathrm{Pin}_{2}$ were prepared that provided optical clarity, good mechanical properties, and the capability of thermal neutron detection. Independent of $\mathrm{B}_{2} \mathrm{Pin}_{2}$ concentration, strong ${ }^{10} \mathrm{~B}$ neutron capture signals around $90 \mathrm{keV}_{\text {ee }}$ were observed at essentially constant light output. Increasing PPO concentration allowed for the use of pulse shape discrimination (PSD) in both fast and thermal neutron detection. High PPO concentrations appear to cause additional alpha quenching that affected the ${ }^{10} \mathrm{~B}$ neutron capture signal. Aging effects after storage in air for several months were observed, which led to degradation of performance and in some samples of mechanical stability.

\section{Introduction}

Detection of neutrons plays an important role in the identification of illicit trafficking of special nuclear materials. With increasing demand, the current state-of-the-art neutron detectors deployed, which are based on ${ }^{3} \mathrm{He}$ gas proportional counters surrounded by thermalizing materials, have become significantly more expensive. Therefore, alternatives with lower cost and 
31 similar efficiency as well as comparable signal to background ratio are sought [1,2]. Recent 32 advances in ${ }^{3} \mathrm{He}$ alternatives have used plastic or liquid scintillator compounds that contain high 33 neutron cross-section isotopes such as ${ }^{6} \mathrm{Li},{ }^{10} \mathrm{~B}$, and ${ }^{155} \mathrm{Gd} /{ }^{157} \mathrm{Gd}$ isotopes [3, 4]. Our group is 34 focusing on the incorporation of boron containing organic compounds because of the significant 35 natural abundance $(\sim 20 \%)$ of ${ }^{10} \mathrm{~B}$, its high thermal neutron reaction cross section and easy 36 availability in highly enriched isotopic concentrations. Previous work incorporating boron in the chemical form of carboranes into plastic matrices achieved promising results [e.g. 5,6,7] and has been used in some commercial products (e.g. Eljen Technology EJ-254). However, the price of the neutron sensitive scintillator product is still dominated by carborane prices and would need to be lowered to allow widespread deployment in large portal type monitor applications. 41 Additionally, the signal to background ratios achieved are not yet competitive to ${ }^{3} \mathrm{He}$ based detectors motivating further research on boron containing scintillator systems.

The neutron induced reaction on ${ }^{10} \mathrm{~B}$ has a thermal cross section of 3837 barns $\left({ }^{3} \mathrm{He}\right.$ is 5333 44 barns) and results in light charged ions (alpha and ${ }^{7} \mathrm{Li}$ ) as the products that carry significant kinetic energy to be easily detected.

$$
\begin{array}{ccc}
{ }^{10} \mathrm{~B}+n_{t h} & \rightarrow & { }^{7} \mathrm{Li}(1.0 \mathrm{MeV})+\alpha(1.8 \mathrm{MeV}) \\
& & Q=2.792 \mathrm{MeV} \quad 7 \% \\
{ }^{10} \mathrm{~B}+n_{t h} & \rightarrow & { }^{7} \mathrm{Li}^{*}(0.83 \mathrm{MeV})+\alpha(1.47 \mathrm{MeV}) \\
& & Q=2.310 \mathrm{MeV} \quad 93 \% \\
{ }^{7} \mathrm{Li}^{*} & \rightarrow & { }^{7} \mathrm{Li}+\gamma(0.48 \mathrm{MeV})
\end{array}
$$

However, organic scintillators suffer in light ion detection from a type of pulse height defect, named alpha quenching, that reduces the number of emitted scintillation photons to a small fraction of the quantity emitted in detection of gamma photons of the same energy. It has been observed that the addition of higher concentrations of additives (fluorescing or neutron sensitive) can lower the overall light output of a scintillator and/or worsen the alpha quenching effect. The combination of light output and alpha quenching determines the overall performance of a scintillator in how well one can separate the neutron reaction signal from gamma backgrounds and electronic noise. 


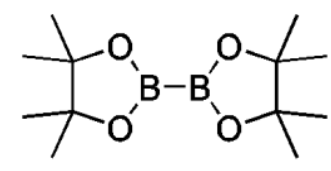

Molecular Weight: 253.94

\section{Elemental Analysis: C, 56.76; H, 9.53; B, 8.51; O, 25.20}

Figure 1: Molecular weight and elemental composition (\%) of bis(pinacolato)diboron $\left(\mathrm{B}_{2} \mathrm{Pin}_{2}\right)$

Bis(pinacolato)diboron $\left(\mathrm{B}_{2} \mathrm{Pin}_{2}\right)$ (Fig. 1) was first used in organic reactions by Miyaura et al to create an intermediate for Suzuki coupling [9]. The conversion of arylhalides to arylboronates using an inexpensive and readily available borylating reagent such as $\mathrm{B}_{2} \mathrm{Pin}_{2}$ has been extensively used in the pharmaceutical and organic electronics industries [10, 11]. Our group uses $\mathrm{B}_{2} \mathrm{Pin}_{2}$ to create high boron content benzene and pyrene derivatives for use as scintillator additives [12]. Before we were able to synthesize the gram quantities necessary for tests with radiation of our new compounds, we ran the series of tests described here with the commercially available precurser $\mathrm{B}_{2} \mathrm{Pin}_{2}(\$ 0.5 / \mathrm{g})$.

All chemicals used for this study were purchased from commercial vendors unless otherwise noted. Vinyl toluene (VT) was purified by removing the inhibitor 4-tert-butylcatechol (TBC) using a plug mixture of basic alumina with a small amount of potassium carbonate. The purified VT was stored in a freezer under nitrogen. Azobisisobutyronitrile (AIBN) radical initiator was recrystallized twice from methanol and stored in a freezer under nitrogen. $\mathrm{B}_{2} \operatorname{Pin}_{2}$ (Matrix Scientific cat. \# 004889) was dried in a Buchi glass oven (model \#B-585) at $40{ }^{\circ} \mathrm{C}$ for $24 \mathrm{~h}$ and stored in a nitrogen filled glovebox. Each scintillator sample was prepared by adding the specific amounts of dopants, AIBN, and vinyl toluene (20 g total) to a $120 \mathrm{ml}(51 \mathrm{~mm}$ dia. x $102 \mathrm{~mm}$ ) glass bottle, followed by a gentle bubbling of argon gas to displace and remove oxygen. The presence of oxygen inhibits polymerization, causes significant light emission quenching, and sample discoloration. The glass bottles were tightly capped and put into a Fischer Scientific Isotemp vacuum oven (model \#280A). The atmosphere inside the vacuum oven was exchanged from air to argon via a few cycles of evacuation followed by argon refill. The sample 
81 polymerization was performed in argon atmosphere over four days at $80^{\circ} \mathrm{C}$ and 12 hours at $90^{\circ} \mathrm{C}$.

82 After polymerization was complete, the glass bottles were broken to provide the raw scintillating polymer "puck". As a first step rough edges were clipped and an even surface produced via machining or hand sanding with medium grid sand paper. Further sanding was performed using finer grit and polishing compounds until finally a buffing wheel achieved a polished surface sufficient for coupling to a photo-detector.

87

\section{Experimental Procedure}

To begin testing, the samples were wrapped tightly with white Teflon tape on all sides except the prepared polished face. This exposed face was then coupled to a photodetector (Hamamatsu PMT assembly, H2431-50) with a thin layer of optical grade silicone grease. The coupled assembly was wrapped in a layer of aluminum foil and sealed to a light-tight condition with black electrical tape. After an optimal voltage $(-2.9 \mathrm{kV}$ bias in all measurements) for viewing relevant features in the output spectra was determined, the PMT was connected to a data acquisition (DAQ) system and biased using standard NIM electronics. The DAQ system includes a $250 \mathrm{Msample/sec}$ waveform digitizer built in-house, and is controlled by a MIDAS software interface [13]. In this approach the voltage pulse as it is recorded from the PMT anode is digitized and stored. The stored pulse is integrated over 2 different integration times where the full integration time period ("Pulse Area") is proportional to the deposited energy in the sample which is converted to scintillation photons. The integral over a delayed time period ("Delayed Area") can be used to describe the pulse shape. The ratio of "Delayed Area" and "Pulse Area" is the "Delayed Area Fraction" and is used later to display and quantify pulse shape discrimination.

Samples were first exposed to a ${ }^{137} \mathrm{Cs}$ source $(1 \mu \mathrm{Ci}$, gamma emission of $662 \mathrm{keV})$ in order to quantify general light output properties. Examining the position of the Compton Edge (C.E.) feature (at $477 \mathrm{keV}$ ) in this output spectra allowed us to calibrate each sample individually and establish an individual sample energy scale (in $\mathrm{keV}_{\mathrm{ee}}$ ). By comparing each sample's C.E. location to a commercial control sample's C.E. location on an absolute scale, we determined a relative light yield output. 
109 Next, the samples were exposed to a ${ }^{244} \mathrm{Cm} /{ }^{13} \mathrm{C}$ source $(160 \mathrm{mCi}$ alpha activity; sealed with neutron and gamma emission) in two different shielding configurations, which produce different mixed neutron and gamma fields. First a "lead castle" setup was used to shield the detector and samples from the gamma flux and transmits a 'fast' neutron spectrum at the detector location, which allowed us to test fast neutron response as well as test and quantify the samples $\mathrm{n} / \gamma$ PSD capabilities. Next the sample and source were placed in a similar castle built out of poly(ethylene) bricks that would alter the neutron spectrum producing a more thermalized flux. This allowed us to test low energy neutron response and identify the ${ }^{10} \mathrm{~B}$ neutron reaction occurring in the sample. Finally, the detector was wrapped in a cadmium shield within the poly(ethylene) cave. Due to the high cadmium thermal neutron capture cross section, this exposure of our samples eliminated the thermal neutron flux and allowed us to collect gamma background spectra that could be subtracted from the non-cadmium shielded spectra, exposing more clearly the samples' response to thermal neutrons. The Cd-shield background subtraction was not performed on the spectra from which we extracted a figure-of-merit (FOM) for pulse shape discrimination.

Once enough statistics were obtained, the collected waveforms were analyzed using the ROOT data analysis framework, to develop relevant output spectra for extracting light output and pulse shape response information [14].

7

\section{Results}

Plastic scintillators generally consist of an inexpensive polymer matrix that absorbs incoming radiation. The matrix becomes "excited" and transfers this energy to fluorescent dopants within the sample that then emit photons in a spectral region matching the photodetector's response function. In order to provide thermal neutron sensitivity, another dopant containing ${ }^{10} \mathrm{~B}$ is added to the formulation; $\mathrm{B}_{2} \mathrm{Pin}_{2}$ in this case. The compatibility of these dopants is essential in creating optically clear, mechanically stable and efficient plastic scintillator samples capable of discriminating neutron signals from a gamma field. We chose the commonly used PPO and POPOP as primary and secondary dopants respectively in order to be able to allow direct comparison with previous literature reports. The initial set of samples (Table 1) was intended to test the solubility limit of $\mathrm{B}_{2} \mathrm{Pin}_{2}$ in PVT, holding the fluorescent dopant concentrations constant. 
139 As $\mathrm{B}_{2} \mathrm{Pin}_{2}$ has a smaller boron content per molecule than carboranes, the achievable boron 140 concentrations are lower than in commercially available products which advertise up to 5\% 141 natural boron content. For comparison, a typical ${ }^{3} \mathrm{He}$ proportional counter with 10 atm gas 142 pressure corresponds to a cross section weighted ${ }^{10} \mathrm{~B}$ content in polymers of app. $0.6 \mathrm{wt} \%$. The 143 increase in $\mathrm{B}_{2} \mathrm{Pin}_{2}$ concentration from 2 to $15 \mathrm{wt} \%$ did not result in significant physical change or 144 a decrease in optical clarity (Figure 6, top). However, some slight nucleation could be seen in the $14515 \mathrm{wt} \%$ sample and when increased to $20 \mathrm{wt} \% \mathrm{~B}_{2} \mathrm{Pin}_{2}(\sim 1.7 \mathrm{wt} \% \mathrm{~B})$, this sample showed 146 significant nucleation which did not allow further machining and testing. Higher dopant 147 concentration usually leads to softening of the polymer matrix, however, this was not noticeable 148 until total dopant concentration was above $15 \%$. Light yield and thermal neutron ${ }^{10} \mathrm{~B}$ capture 149 results are detailed below in Figures 2-3.

150

151 Table 1: Optimizing boron additive in PVT

\begin{tabular}{|c|c|c|c|c|c|c|c|c|}
\hline \multirow{2}{*}{ Sample* } & \multirow{2}{*}{ Matrix } & \multicolumn{2}{|l|}{ Primary dopant } & \multicolumn{2}{|c|}{ Wavelength shifter } & $\begin{array}{c}\mathrm{B}_{2} \mathrm{Pin}_{2} \\
\text { content }\end{array}$ & $\begin{array}{c}\text { Nat-B } \\
\text { content }\end{array}$ & ${ }^{10} \mathrm{~B}$ content \\
\cline { 3 - 9 } & & Name & $\mathrm{wt} \%$ & Name & $\mathrm{wt} \%$ & $\mathrm{wt} \%$ & $\mathrm{wt} \%$ & $\mathrm{wt} \%$ \\
\hline 1 & PVT & PPO & 1 & POPOP & 0.1 & - & - & - \\
\hline 2 & PVT & PPO & 1 & POPOP & 0.1 & 2 & 0.17 & 0.03 \\
\hline 3 & PVT & PPO & 1 & POPOP & 0.1 & 5 & 0.43 & 0.09 \\
\hline 4 & PVT & PPO & 1 & POPOP & 0.1 & 10 & 0.85 & 0.17 \\
\hline 5 & PVT & PPO & 1 & POPOP & 0.1 & 11.75 & 1 & 0.2 \\
\hline 6 & PVT & PPO & 1 & POPOP & 0.1 & 15 & 1.28 & 0.25 \\
\hline 7 & PVT & PPO & 1 & POPOP & 0.1 & 20 & 1.7 & 0.34 \\
\hline
\end{tabular}

152

*Total mass of each sample is app. $20.0 \mathrm{~g}$ 


\section{${ }^{137}$ Cs Compton Edge Comparison}

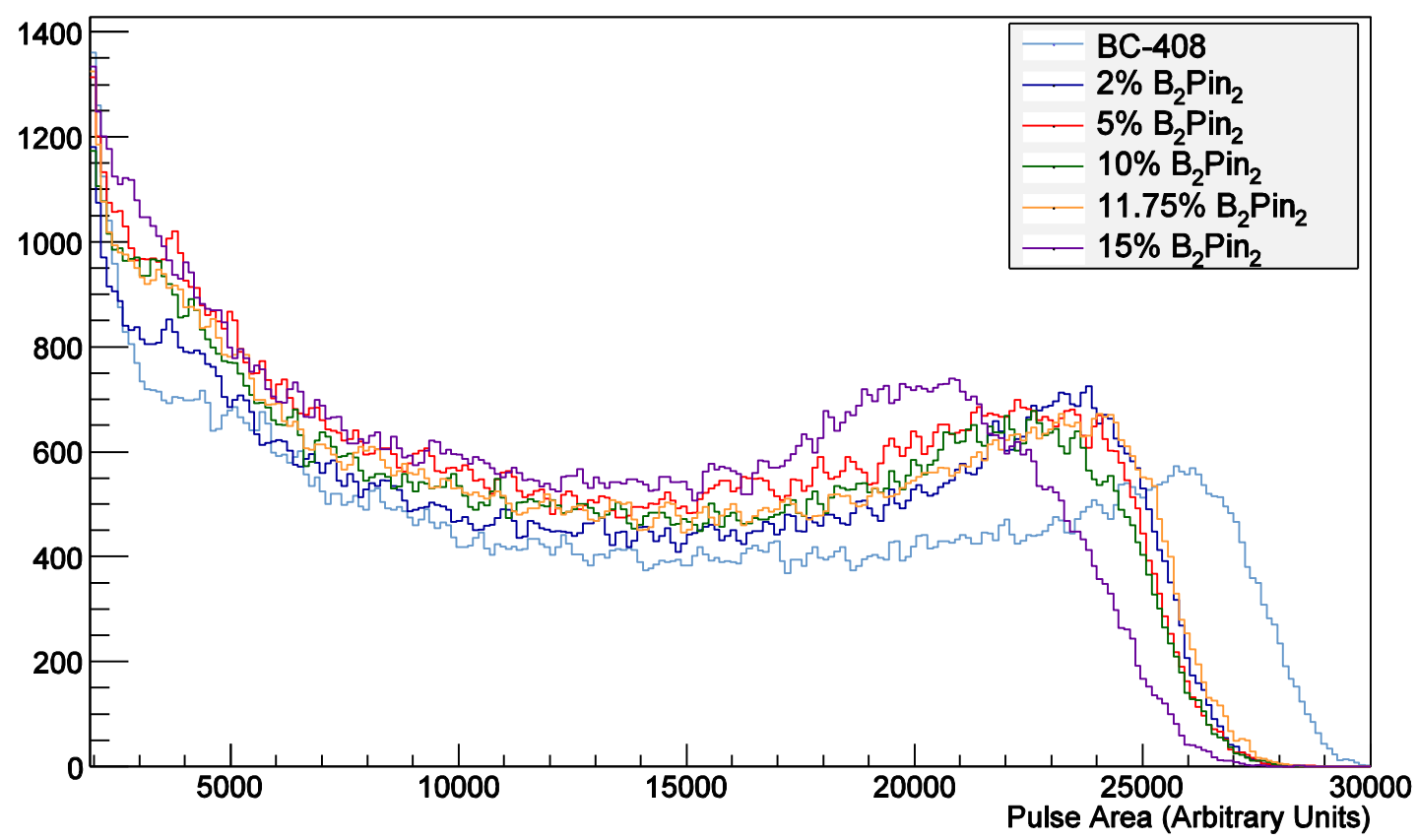

153

154

155

156

157 The $\mathrm{B}_{2} \mathrm{Pin}_{2}$ concentration appears to have little effect on light output when added at the $10 \mathrm{wt} \%$

158

159

160

161

162

163

164

Figure 2: Number of counts vs uncalibrated pulse area in scintillating PVT based samples as measured through exposure to a ${ }^{137} \mathrm{Cs} \gamma$-source using the same PMT bias value.

level as shown in Figure 2; most samples produce a Compton Edge (numerically characterized as the halfway point between Compton edge maximum and zero) at $92-94 \%$ that of a commercial control sample (Saint Gobain BC-408; 10,000 photons/1 MeV e-). There is a more significant decrease at $15 \mathrm{wt} \%$ which is suspected to be due to the approaching solubility limit of $\mathrm{B}_{2} \mathrm{Pin}_{2}$. It should be noted that in the few cases where we repeated samples of the same composition, our measured light yields varied of order $\pm 2-3 \%$-points. Therefore, we would characterize sample variation in the range of $92-94 \%$ as essentially constant. 


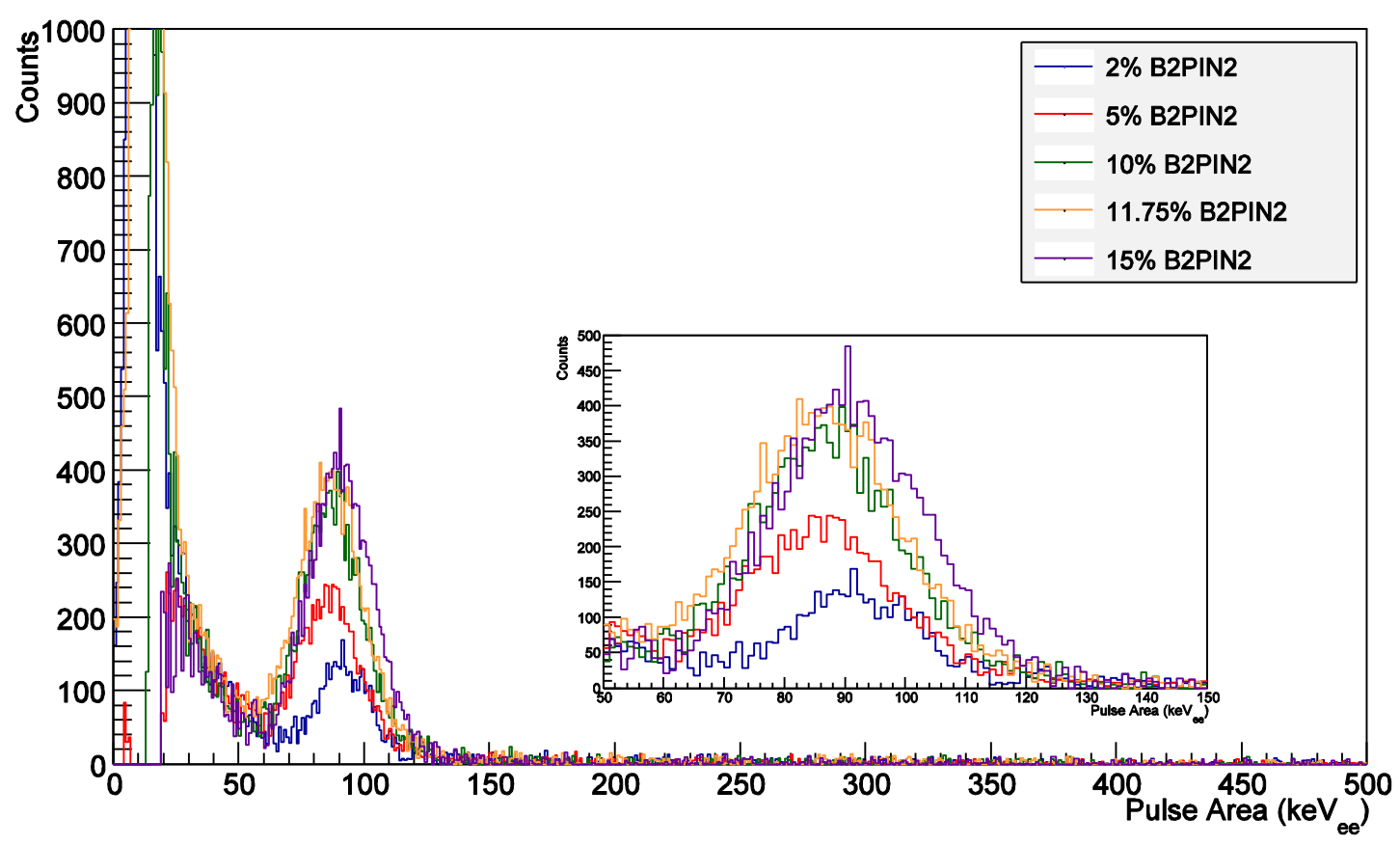

165

166

167

168

169

170

171

172

173

174

175

176

177

178

179

180

181

182

183

Figure 3: Number of counts versus calibrated pulse area $\left(\mathrm{keV}_{\mathrm{ee}}\right)$ for ${ }^{10} \mathrm{~B}$ thermal neutron reaction spectra with gamma response subtracted via cadmium shielded measurement. The $\mathrm{keV}_{\text {ee }}$ sample scale adjusts all samples to the ${ }^{137} \mathrm{Cs}$ Compton edge position of each sample (light yield adjustment) to make changes in alpha quenching apparent.

Thermal neutrons in the ${ }^{244} \mathrm{Cm} /{ }^{13} \mathrm{C}$ "Poly Cave" setup were captured by the naturally occurring ${ }^{10} \mathrm{~B}$ isotope within $\mathrm{B}_{2} \mathrm{Pin}_{2}$. As described earlier, although the reaction produces light ions with kinetic energies in the $\mathrm{MeV}$ range, the signal registered in the detector samples was, due to alpha quenching, around $90 \mathrm{keV}_{\mathrm{ee}}$, only slightly varying with $\mathrm{B}_{2} \mathrm{Pin}_{2}$ concentration. In order to extract the neutron capture peaks clearly, the cadmium shielded spectra were subtracted from the "poly cave" capture spectra as shown in Figure 3. The results here reported on the position $\left(\mathrm{keV}_{\mathrm{ee}}\right)$ of the ${ }^{10} \mathrm{~B}$ neutron capture peak are similar to previously reported values on boron loaded plastic scintillators using carboranes [5,7]. As mentioned above, raising the $\mathrm{B}_{2} \mathrm{Pin}_{2}$ concentration appears to produce an essentially constant light output and alpha quenching. This indicates a good match of the neutron sensitive dopant to the plastic matrix in terms of scintillation relevant properties. 
Table 2: Plastic compositions with over-doped PPO and 1\% "boron” additive

\begin{tabular}{|c|c|c|c|c|c|c|c|}
\hline Sample* & \multirow{2}{*}{ Matrix } & \multicolumn{2}{|c|}{ Primary dopant } & \multicolumn{2}{|c|}{ Wavelength shifter } & $\mathrm{B}_{2} \mathrm{Pin}_{2}$ & DVB \\
\cline { 3 - 8 } & & Name & $\mathrm{wt} \%$ & Name & $\mathrm{wt} \%$ & $\mathrm{wt} \%{ }^{*}$ & $\mathrm{wt} \%$ \\
\hline 1 & PVT & PPO & 10 & POPOP & 0.1 & 11.75 & - \\
\hline 2 & PVT & PPO & 15 & POPOP & 0.1 & 11.75 & 0.1 \\
\hline 3 & PVT & PPO & 20 & POPOP & 0.1 & 11.75 & 0.1 \\
\hline 4 & PVT & PPO & 25 & POPOP & 0.1 & 11.75 & 0.1 \\
\hline
\end{tabular}

$* 11.75 \% \mathrm{~B}_{2} \mathrm{Pin}_{2}$ is equivalent to $1 \%$ boron content.

187 A second set of samples was prepared and tested with the intent of achieving PSD in our samples

188

189

190

191

192

193

194

195

196

197

198

199

200

201

202

203

204

205

206

207

208

209

210 using the scintillating fluor over-doping method originally developed by Brooks et al. [15] which in recent years inspired further work in several laboratories $[16,17,18]$. The compositions used in this sample set are provided in Table 2. The $11.75 \mathrm{wt} \% \mathrm{~B}_{2} \mathrm{Pin}_{2}$ concentration is equivalent to 1 wt\% natural boron content, and was chosen to be below the solubility limit of the $\mathrm{B}_{2} \mathrm{Pin}_{2} / \mathrm{PVT}$ system. The plastic samples were polished and prepared as described earlier and their detector response properties analyzed with the same radioactive source configurations. In our tests significant PSD was not quantifiable until the sample contains at least 10\% PPO. Meaningful separation between neutrons and gammas in our energy range of interest (of order $100 \mathrm{keV}_{\mathrm{ee}}$ ) was not seen until higher concentrations $(\sim 20 \%)$. The $\mathrm{B}_{2} \mathrm{Pin}_{2}$ content, combined with the high amount of PPO necessary to achieve PSD, acts as "impurities" in the polymerization process likely leading to lower polymer molecular weight and thus diminished mechanical properties. In order to address this concern, divinylbenzene (DVB), a crosslinking agent, was introduced into the sample solution prior to polymerization. With the crosslinking agent added, the plastic sample containing $20 \mathrm{wt} \%$ PPO $0.1 \mathrm{wt} \%$ POPOP and $11.75 \mathrm{wt} \% \mathrm{~B}_{2} \mathrm{Pin}_{2}$ was at a "hardness" level that could be cut and prepared for our standard detector test. The Compton Edge of the ${ }^{137} \mathrm{Cs}$ spectrum was $98 \%$ light output of our commercial plastic scintillator (Saint Gobain Crystals, BC-408). However, the PPO over-doping method appeared to worsen the alpha quenching significantly and the ${ }^{10} \mathrm{~B}$ thermal neutron capture peak was located at app. $60 \mathrm{keV}_{\text {ee }}$ The resulting spectrum from the thermalized ${ }^{244} \mathrm{Cm} /{ }^{13} \mathrm{C}$ neutron source is detailed in Figure 4 and various PSD figures of merit (FoM) numbers using the fast neutron spectrum are shown in Figure 5. The measured FoM at $100 \mathrm{keV}_{\text {ee }}$ of 1.04 compares well to the published value of 0.9 at $120 \mathrm{keV}_{\text {ee }}$ for the commercial Eljen Technology EJ-299-33, which presumably uses the PPO over-doping method [19]. 


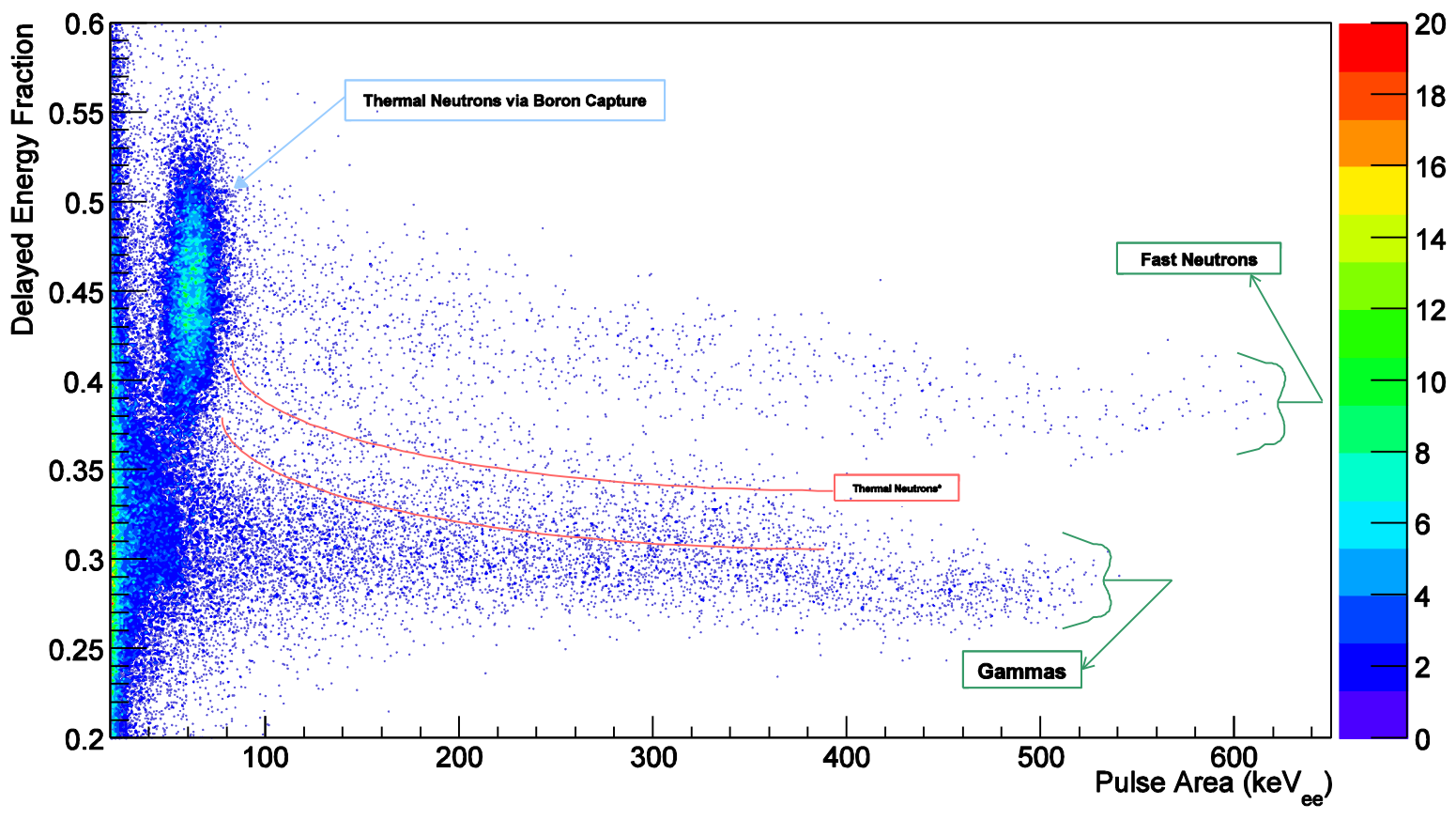

211

212 Figure 4: Full PSD (Full Pulse Area over Delayed Area Fraction) spectrum of PVT sample 213 containing $11.75 \mathrm{wt} \%$ of $\mathrm{B}_{2} \mathrm{Pin}_{2}, 20 \mathrm{wt} \%$ PPO, $0.1 \mathrm{wt} \%$ POPOP as measured in the ${ }^{244} \mathrm{Cm} /{ }^{13} \mathrm{C}$ 214 "poly cave" setup. In addition to the thermal neutron capture peak a band between the fast 215 neutron and gamma bands becomes tentatively visible which contains also thermal neutron 216 capture events, however, here the exited state of the ${ }^{7} \mathrm{Li}$ reaction product is populated and both an 217 alpha and a $480 \mathrm{keV}$ gamma is recorded in coincidence. 


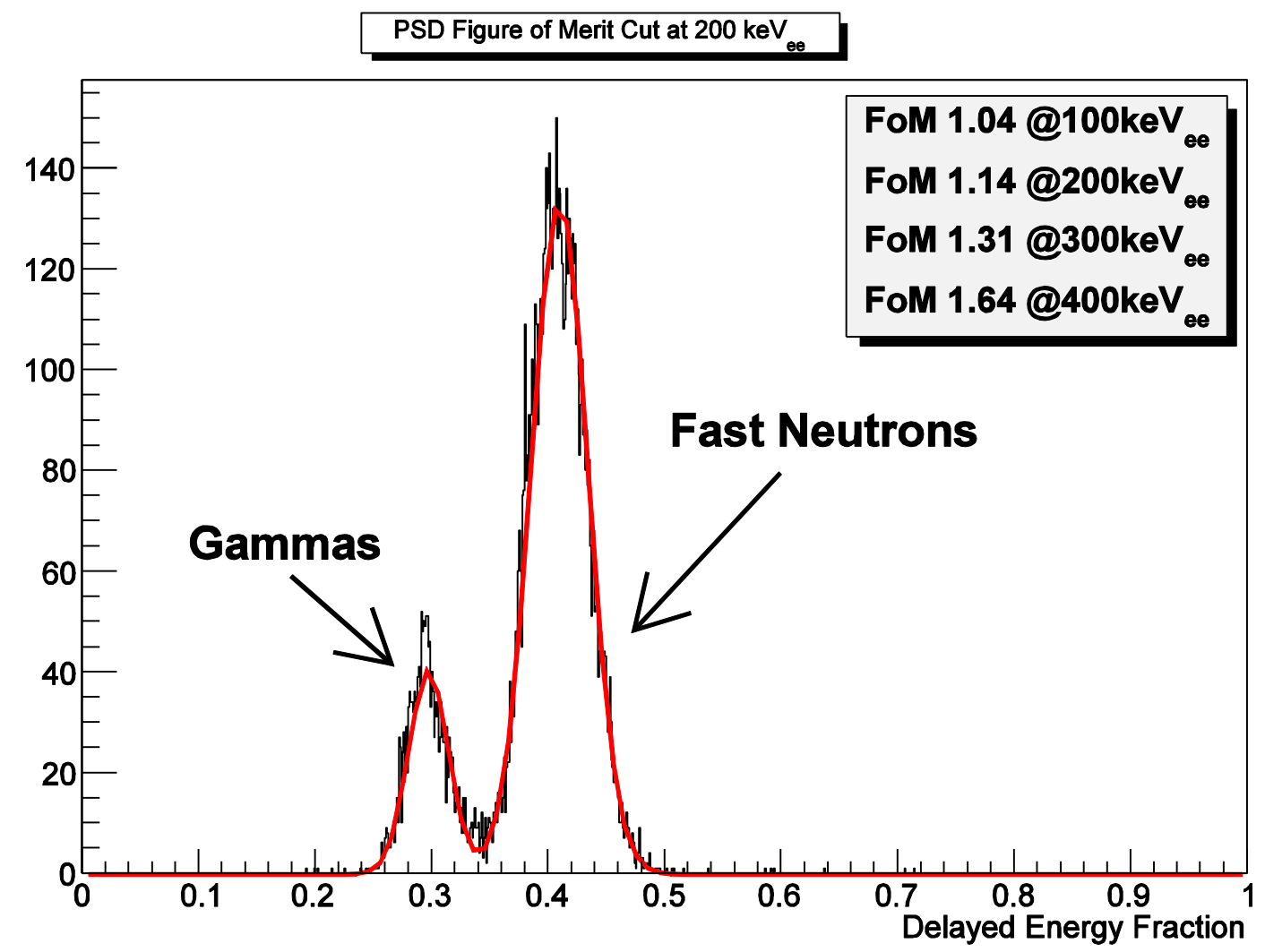

219

220

221

222

223

224

225

226

227

228

229

230

231

232

233

Figure 5: PSD Figure of Merit values resulting from various energy cuts (spectrum shown from $200 \mathrm{keV}_{\mathrm{ee}}$ cut) using the same sample as in Fig. 4 in the "lead cave" setup and projecting the Full PSD Spectrum after the energy cut onto the y-axis.

In order to assess the aging effects, we retested the scintillator samples 9 months later after storage in ambient conditions without humidity control. There was some slight visual yellowing/clouding of the $\mathrm{B}_{2} \mathrm{Pin}_{2}$ doped samples (e.g. Fig. 6, bottom) with average decrease of approximately $20 \%$ in light yield. However, ${ }^{10} \mathrm{~B}$ thermal neutron reaction signals could still be detected from in the samples containing PPO at the $1 \mathrm{wt} \%$ level. The over-doped PPO samples while still displaying $\mathrm{n} / \gamma$ PSD, had turned opaque with negligible light output $(\sim 10 \%$ of $\mathrm{BC}$ 408). The physical change in our sample is likely due to oxidation of residual radicals left from the radical bulk polymerization method used in our production process. Future work will need to be devoted to employ radical quenchers, antioxidants or thermal annealing procedures to achieve the long ( years) lifetimes expected from plastic scintillator products. 

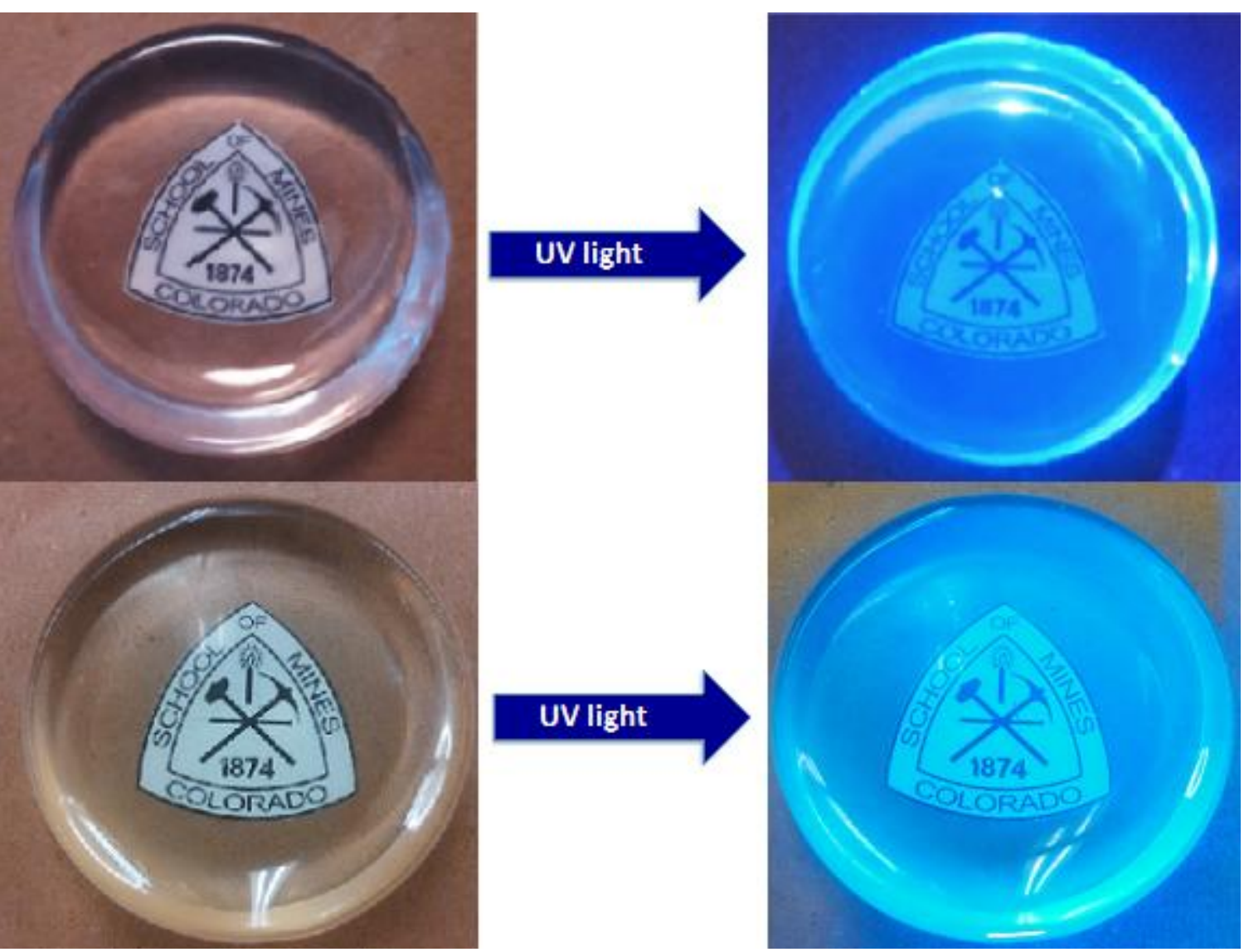

235 Fig. 6: Plastic scintillator sample containing $1 \mathrm{wt} \%$ PPO, 0.1\% POPOP and $11.75 \% \mathrm{~B}_{2} \mathrm{Pin}_{2}$ in PVT at the time of first testing (top) and after 9 months ambient storage (bottom).

The research described here demonstrates that $\mathrm{B}_{2} \mathrm{Pin}_{2}$ can be used as a low cost ${ }^{10} \mathrm{~B}$ additive that is compatible with commonly used plastic scintillator formulations to achieve plastic scintillator detectors with thermal neutron sensitivity. $\mathrm{B}_{2} \mathrm{Pin}_{2}$ concentrations up to the solubility limit have

243 little effect on sample light yield and alpha quenching, allowing detectors to be produced with on 244 the order of $1 \mathrm{wt} \%$ natural boron content. Thermal neutrons are easily detected with the ${ }^{10} \mathrm{~B}$ neutron capture peak occurring at $\sim 90 \mathrm{keV}_{\mathrm{ee}}$ in the basic formulations, and at $\sim 60-65 \mathrm{keV}_{\text {ee }}$ in

246 formulations with PPO overdoping to achieve PSD. In the PSD samples, analysis allowed for 247 separation of gamma and proton (or alpha) signals down to low energies providing an additional 248 factor to distinguish the thermal neutron signal. Further work is necessary to suppress aging 
effects through improvements in the scintillator formulation and/or production process. The measurements here were performed using $\mathrm{B}_{2} \mathrm{Pin}_{2}$ with natural boron isotopic distribution, but pathways of synthesizing boron containing organic additives from readily available ${ }^{10} \mathrm{~B}$ enriched compounds are being investigated by our group.

\section{Acknowledgements}

This research was funded through US DoD DTRA grant \# HDTRA1-11-1-0025 and US DHS DNDO grant \# DHS-14-DN-077-AR-NC7. The authors would like to thank Dr. Peter Menge and Dr. Michael Kusner from Saint Gobain Crystals for helpful discussions in this study.

\section{References}

1. Kouzes, R.T., et al., Nuclear Instruments and Methods in Physics Research Section A: Accelerators, Spectrometers, Detectors and Associated Equipment 623 (2010) 1035

2. Peurrung, A.J., Nuclear Instruments and Methods in Physics Research Section A: Accelerators, Spectrometers, Detectors and Associated Equipment 443 (2000) 400

3. Kouzes, R.T., A.T. Lintereur, and E.R. Siciliano, Nuclear Instruments and Methods in Physics Research Section A: Accelerators, Spectrometers, Detectors and Associated Equipment 784 (2015) 172

4. Bertrand, G.H.V., M. Hamel, and F. Sguerra, Chemistry-A European Journal 20 (2014) 15660

5. Pawełczak, I., et al., Nuclear Instruments and Methods in Physics Research Section A: Accelerators, Spectrometers, Detectors and Associated Equipment 751 (2014) 62

6. D.M. Drake, W.C. Feldman and C. Hurlbut, Nuclear Instruments and Methods in Physics Research Section A: Accelerators, Spectrometers, Detectors and Associated Equipment 247 (1986) 576

7. K.J. Riley et al., IEEE Nuclear Science Symposium Conference Record (2010) 1777

8. Mughabghab, S.F., Atlas of Neutron Resonances: Resonance Parameters and Thermal Cross Sections. $Z=1-1005^{\text {th }}$ Edition, Elsevier, Amsterdam (2006)

9. $\quad$ Miyaura, N. and A. Suzuki, Chemical Reviews 95 (1995) 2457 
287 10. Kotha, S., K. Lahiri, and D. Kashinath, Tetrahedron 58 (2002) 9633

289 11. Torborg, C. and M. Beller, Advanced Synthesis \& Catalysis 351 (2009) 3027

12. Yemam, H.A., et al., Scientific Reports 5 (2015) 13401

13. Ritt, S. and P. Amaudruz, https://midas.triumf.ca/MidasWiki/index.php/Main_Page accessed 11/1/2015

14. Brun, R. and F. Rademakers, Nuclear Instruments and Methods in Physics Research Section A: Accelerators, Spectrometers, Detectors and Associated Equipment 389 (1997) 81

15. F.D. Brooks, R.W. Pringle and B.L. Funt, IRE Transactions on Nuclear Science NS-7 (1960) 35

16. J.K. Walker, Y. Noh and R.T. Farley, Nanoptics Inc. Patent \#8993968 (2012)

17. Zaitseva, N., et al., Nuclear Instruments and Methods in Physics Research Section A: Accelerators, Spectrometers, Detectors and Associated Equipment 668 (2012) 88

18. P. Blanc et al., Nuclear Instruments and Methods in Physics Research Section A: Accelerators, Spectrometers, Detectors and Associated Equipment 750 (2014) 1

19. S.A. Pozzi, M.M. Bourne and S.D. Clarke, Nuclear Instruments and Methods in Physics Research Section A: Accelerators, Spectrometers, Detectors and Associated Equipment 723 (2013) 19 